\title{
Multiwavelength observation of a large-scale flux rope eruption above a kinked small filament ${ }^{\star}$
}

\author{
Pankaj Kumar and Kyung-Suk Cho
}

\author{
Korea Astronomy and Space Science Institute (KASI), 305-348 Daejeon, Republic of Korea \\ e-mail: pankaj@kasi.re.kr
}

Received 18 December 2013 / Accepted 25 September 2014

\begin{abstract}
We analyzed multiwavelength observations of a western limb flare (C3.9) that occurred in AR NOAA 111465 on 30 April 2012. The high-resolution images recorded by SDO/AIA 304, $1600 \AA$ and Hinode/SOT H $\alpha$ show the activation of a small filament (rising speed $\sim 40 \mathrm{~km} \mathrm{~s}^{-1}$ ) associated with a kink instability and the onset of a C-class flare near the southern leg of the filament. The first magnetic reconnection occurred at one of the footpoints of the filament and caused the breaking of its southern leg. The filament shows unwinding motion of the northern leg and apex in counterclockwise direction and failed to erupt. A flux-rope structure (visible only in hot channels, i.e., AIA 131 and $94 \AA$ and Hinode/SXT) appeared along the neutral line during the second magnetic reconnection that occurred above the kinked filament. The formation of the RHESSI hard X-ray source $(12-25 \mathrm{keV})$ above the kinked filament and the simultaneous appearance of the hot $131 \AA$ loops associated with photospheric brightenings (AIA $1700 \AA$ ) indicates the particle acceleration along these loops from the top of the filament. In addition, extreme ultraviolet disturbances or waves observed above the filament in $171 \AA$ also show a close association with magnetic reconnection. The flux rope rises slowly $\left(\sim 100 \mathrm{~km} \mathrm{~s}^{-1}\right)$, which produces a very large twisted structure possibly through reconnection with the surrounding sheared magnetic fields within 15-20 min, and showed an impulsive acceleration reaching a height of about 80-100 Mm. AIA 171 and SWAP $174 \AA$ images reveal a cool compression front (or coronal mass ejection frontal loop) surrounding the hot flux rope structure.
\end{abstract}

Key words. Sun: flares - Sun: filaments, prominences - Sun: coronal mass ejections (CMEs) - Sun: magnetic fields - molecular data

\section{Introduction}

A magnetic flux rope is a coherent helical structure that contains twisted field lines wrapped around its guiding axis. A flux rope is a crucial part of a coronal mass ejection (CME) and plays an important role in the onset of solar eruptions and associated flares. The CME models involve the eruption of a flux rope either by magnetic reconnection or by magnetohydrodynamic (MHD) instabilities. For example, an emerging flux trigger (Chen \& Shibata 2000), flux cancellation (Amari et al. 2003a,b, 2010), tether cutting (Moore et al. 2001), and breakout models (Antiochos et al. 1999; Lynch et al. 2004; Karpen et al. 2012) require magnetic reconnection below or above the flux rope. On the other hand, numerical MHD simulations of the kink instability suggest that if the twist of the flux rope exceeds a critical value ( $\sim 1.75$ field line turns), then it becomes unstable (Fan \& Gibson 2003, 2004; Kliem et al. 2004; Török \& Kliem 2003; Török et al. 2004). Alternatively, the decrease of an overlying magnetic field $(B)$ with height $(H$; decay in$\operatorname{dex} n=-\mathrm{d}(\log B) / \mathrm{d}(\log H)>1.5$, condition for torus instability) above the eruption site alone can decide whether the eruption of a flux rope is successful or fails (Kliem \& Török 2006; Aulanier et al. 2010; Démoulin \& Aulanier 2010; Olmedo \& Zhang 2010). In addition, the interaction of filaments or flux ropes caused by the rotation of sunspots is also found to be associated with the CME onset (Kumar et al. 2010, 2013; Török et al. 2011, 2013; Yan et al. 2012). For the detailed mechanisms and

\footnotetext{
* Movies associated with Figs. 2 and 7 are available in electronic form at http://www. aanda.org
}

instabilities involved in solar eruptions, see the recent reviews by Chen (2011), Webb \& Howard (2012), and Aulanier (2014).

The helical structure of the flux rope has been inferred from observations made with the SOHO/LASCO (Solar and Heliospheric Observatory/Large Angle and Spectrometric Coronagraph) coronagraph and interplanetary scintillation (IPS; e.g., Dere et al. 1999; Manoharan 2010). The cylindrical or circular features observed in the coronagraph images support the assumption about the flux rope topology in projection (see, Vourlidas et al. 2013; Vourlidas 2014). The flux rope is recognized as a magnetic cloud in solar wind observations at $1 \mathrm{AU}$ from the low plasma beta $(\beta)$ and the smooth rotation of the magnetic field vector $\left(\boldsymbol{B}_{z}\right)$ (Burlaga et al. 1982; Bothmer \& Schwenn 1998; Kumar et al. 2011a; Marubashi et al. 2012). In the solar atmosphere, the filament plasma is considered to be supported in the dips of a helical flux rope (Priest et al. 1989; Su et al. 2011). In addition, a twisted flux rope structure is sometime observed co-spatially in soft X-ray and extreme ultraviolet (EUV) channels (hot and cool channels) above the neutral line (Kumar et al. 2011b). Recently, flux ropes have been observed in the AIA hot channels (131 and $94 \AA, T \sim 8 \mathrm{MK}$ ), which are elongated Sshaped structures (Zhang et al. 2012; Patsourakos et al. 2013; Cheng et al. 2014). Expansion and rise of these structures are associated with the formation of CMEs in the low corona.

Most of the CME models require the presence of the flux rope before the eruption (e.g., flux emergence, flux cancellation, MHD instability related models), while other models (including breakout and tether-cutting models) consider that the flux rope is formed (above the neutral line) during magnetic reconnection (Gosling et al. 1995; Longcope \& Beveridge 2007). The details 
of the flux rope formation in the low corona is poorly understood. It is debated whether the flux rope is present well before the eruption or forms by magnetic reconnection during the eruption. A model of solar prominences indicates that the prominence plasma is supported inside the twisted flux tube (Priest et al. 1989; van Ballegooijen et al. 2000), and this twisted flux tube is formed by the flux cancellation in a sheared arcade (van Ballegooijen \& Martens 1989). Alternatively, the helical magnetic field of the filament is explained by the twisted flux rope model (Rust \& Kumar 1994), in which the flux tube emerges (already twisted) through the photosphere supporting the filament plasma. The numerical MHD simulation by Fan (2001) indicated that the emergence process stops when the flux rope reaches the photosphere and forms a sheared arcade in the corona. Gary \& Moore (2004) and Kumar et al. (2012) have provided evidence of three to four helical turns within or below the prominence, which supports the flux rope model. Okamoto et al. (2008) showed evidence of the emergence of a helical flux rope below an active region prominence. On the other hand, Gosling et al. (1995) suggested that magnetic reconnection occurring within the leg of the outward moving magnetic loops causes the formation of the helical flux rope structure. Qiu et al. (2007) quantitatively compared the total magnetic reconnection flux in the low corona (measured from flare ribbons) in the wake of CMEs and the magnetic flux in magnetic clouds, favoring the flux rope formation during magnetic reconnection. The model developed by Longcope \& Beveridge (2007) also explains the flux rope formation by a sequence of magnetic reconnection above the polarity inversion line in a two-ribbon flare.

Soft X-ray images often show sigmoid structures (S- and inverse S-shaped; Canfield et al. 1999; Pevtsov 2002) with a twisted magnetic flux rope topology (Rust \& Kumar 1996; Manoharan et al. 1996). Forward and reverse S-shaped sigmoids are generally (not all) observed in the southern and northern hemisphere, with the positive (right-handed) and negative (left-handed) helicity according to the hemispheric helicity rule (Pevtsov et al. 1995; Rust \& Kumar 1996). Gibson et al. (2006) explained the evolving sigmoid as evidence of a long-lived coronal flux rope before, during, and after a CME eruption. The tether-cutting model (Moore et al. 2001) explains the flux rope formation by magnetic reconnection in between two opposite $J$ shaped elbow structures (arcade loops) that form a continuous S-shaped loop and an underlying loop as a result of magnetic reconnection. In the model developed by Aulanier et al. (2010), the flux rope formation takes place slowly because of photospheric bald patch and coronal slip-running tether-cutting reconnection. Furthermore, a few studies (using Hinode/SXT and SOHO/MDI data) suggested a gradual transformation of the arcade field into a sigmoid or flux rope as a result of photospheric flux cancellation over extended periods before the onset of an eruption (Green \& Kliem 2009; Tripathi et al. 2009; Green et al. 2011).

Using multiwavelength observations from TRACE, STEREO, and Hinode/SXT, Kumar et al. (2011b) provided an example of the presence of a helical flux rope before and during the solar eruption at multitemperature plasma. Recent AIA observations have also revealed the formation of a hot flux rope (visible in AIA 94 and $131 \AA$ ) during the flare impulsive phase on 3 November 2010 (Cheng et al. 2011, 2012), which was followed by magnetic breakout (Kumar \& Innes 2013). Moreover, some of the AIA observations suggest the formation of a hot flux rope well before the solar eruption (Zhang et al. 2012; Patsourakos et al. 2013; Kumar \& Cho 2013). However, the exact mechanism of the flux rope formation, eruption (i.e.,

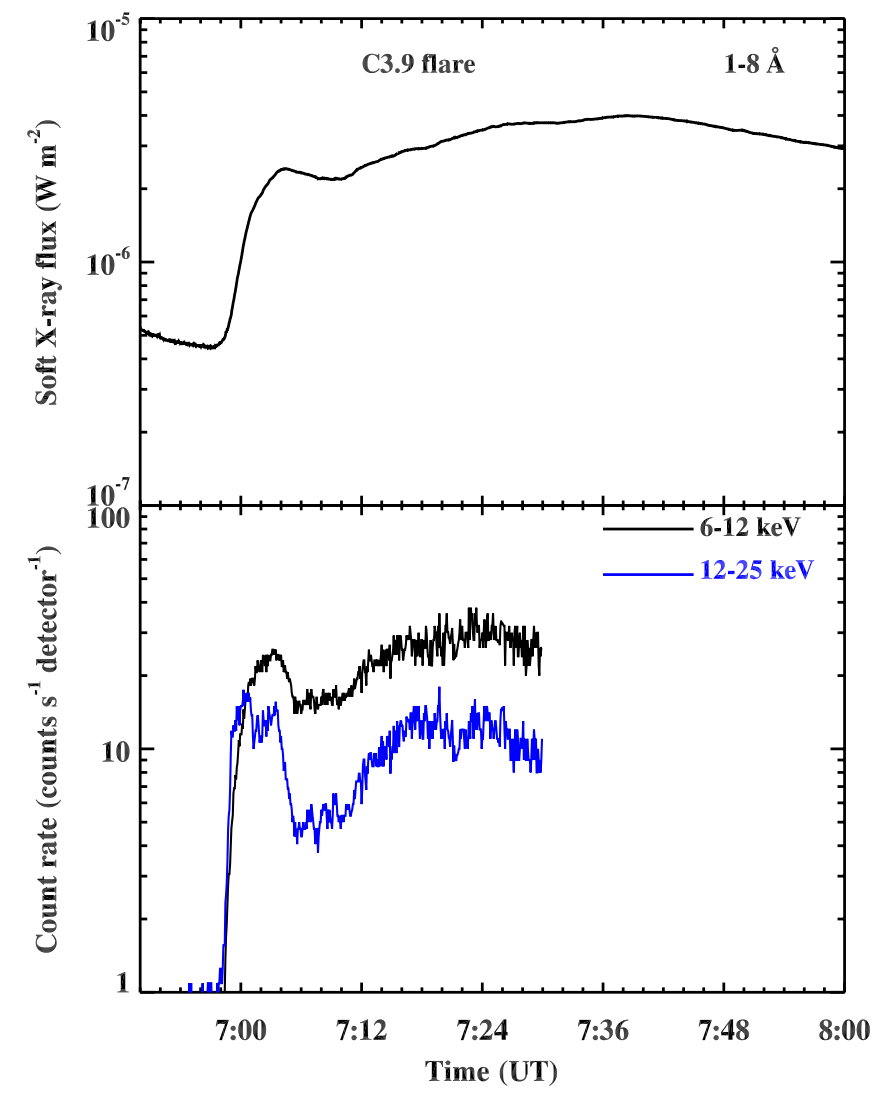

Fig. 1. GOES soft X-ray flux in the $1-8 \AA$ channel (top) and RHESSI hard X-ray flux profiles in the $6-12 \mathrm{keV}$ and $12-25 \mathrm{keV}$ channels (bottom) for the C3.9 flare on 30 April 2012.

trigger), and their relationship with the filament structures is not well known yet.

At present, high-resolution multiwavelngth data from SDO/AIA and Hinode provide an opportunity to investigate the onset mechanism of the solar eruptions and associated phenomena in great detail. In this paper, we present multiwavelength observations of a flux rope eruption followed by a C-class flare that occurred on 30 April 2012. AIA observations combined with RHESSI hard X-ray images reveal the appearance of a flux rope above the small filament during magnetic reconnection. So far, the appearance of a hot flux rope structure above a kinked small filament has not been reported in earlier data sets. In Sect. 2, we present the multiwavelength observational data sets and in the last section, we discuss the results.

\section{Observations and results}

The Atmospheric Image Assembly (AIA; Lemen et al. 2012) onboard the Solar Dynamics Observatory (SDO; Pesnell et al. 2012) obtains full-disk images of the Sun (field of view $\sim 1.3 R_{\odot}$ ) with a spatial resolution of $1.5^{\prime \prime}\left(0.6^{\prime \prime}\right.$ pixel $\left.^{-1}\right)$ and a cadence of $12 \mathrm{~s}$ in ten EUV and UV filters. For this study, we used $171 \AA$ (Fe IX, $T \sim 0.7 \mathrm{MK}), 131 \AA$ (Fe VIII/XXI/XXIII, $T \sim 0.4,11$ and $16 \mathrm{MK}), 94 \AA$ (Fe XVIII, $T \sim 6.3 \mathrm{MK}), 335 \AA$ (Fe XVI, $T \sim 2.5 \mathrm{MK}), 304 \AA$ (He II, $T \sim 0.05 \mathrm{MK}), 1600 \AA$ (CIV and continuum, $T \sim 0.1 \mathrm{MK}$ ), and 1700 (continuum, $T \sim$ $5000 \mathrm{~K}) \AA$ images. The active region NOAA 11465 , located at the western limb, produced a C3.9 flare on 30 April 2012. This flare was a long-duration event that started at $\sim 06: 56 \mathrm{UT}$, peaked at $\sim 07: 38 \mathrm{UT}$, and ended at $\sim 08: 19$ UT. Figure 1 displays the GOES soft X-ray flux profile in the $1-8 \AA$ channel 
(top) and RHESSI hard X-ray flux profiles (bottom) in the 6-12 keV (black) and 12-25 keV (blue) energy bands. The hard X-ray fluxes indicate two stages of energy release during (i) 06:58-07:05 UT; and (ii) 07:13-07:30 UT.

\subsection{Filament eruption and associated flare}

To see the evolution of the flare onset in the lower solar atmosphere (i.e., chromosphere and transition region), we used the AIA $304 \AA$ images. Figure 2 shows some of the selected images of the flare site just before the impulsive phase and during the flare. We observe a small filament at the flare site at 06:57:20 UT (Fig. 2a). The brightening starts below the southern leg of the filament at 06:58:08 UT, and the filament started to rise. Figure $2 \mathrm{c}$ shows the kinked filament with two legs (marked by $\mathrm{N}$ and $\mathrm{S}$ ). The southern (S) leg of the filament breaks, possibly as a result of reconnection, and it shows unwinding motion at the apex and counterclockwise rotation of the northern leg $(\mathrm{N})$ (see Figs. 2d, f). The direction of the rotation is marked by blue arrows. This sense of rotation is detected by the bright and dark treads of the filament. The apex of the filament breaks and falls back (i.e., failed eruption) to the solar surface (Fig. $2 \mathrm{~g}$ ). The temporal evolution of the filament (unwinding motion with failed eruption) in $304 \AA$ is shown in the animation attached to Fig. 2. To determine the rising speed of the filament and its relation with the flare onset, we plot the intensity distribution along the slice A, which is shown by the dotted line in Fig. 2f. The stack plot is shown in Fig. 2h. The average intensity of the eruption site (in AIA $304 \AA$ ) is plotted with a blue curve. The rising of the filament and associated brightening in $304 \AA$ occurred simultaneously. From the linear fit, the estimated average speed of the filament was $\sim 40 \mathrm{~km} \mathrm{~s}^{-1}$.

We used the differential affine velocity estimator (DAVE; Schuck 2006) method to track the apparent plasma flows (in the plane of sky) in and around the filament. Coaligned pairs of the AIA images in $304 \AA$ (with 12 sec cadence) were used to deduce the plasma flows. Figures $2 \mathrm{i}-\mathrm{k}$ display the AIA $304 \AA$ images overlaid by flow fields during the filament eruption. The flow vectors are in the upward direction along the northern leg, and in the southward direction at the apex. The direction of the flow vectors agrees with the expansion and rotation of the northern leg and apex of the filament in the counterclockwise direction. The longest arrows represent flow speeds of 204, 178, and $158 \mathrm{~km} \mathrm{~s}^{-1}$ in panels (i), (j), and (k) respectively, which are larger than the flow speeds observed in the tornado-like prominence (55-95 $\mathrm{km} \mathrm{s}^{-1}$ ) computed by Li et al. (2012) using the local correlation-tracking (LCT) method.

To see the chromospheric evolution of the filament in a better spatial resolution than AIA, we also used the high-resolution $\left(0.08^{\prime \prime}\right.$ pixel $\left.^{-1}\right) \mathrm{H} \alpha(6563 \AA)$ images observed by the Solar Optical Telescope (SOT; Tsuneta et al. 2008; Suematsu et al. 2008) onboard the Hinode satellite (Kosugi et al. 2007). Figure 3 displays a series of $\mathrm{H} \alpha$ images showing the evolution of the small filament and associated flare. Figure 3 a shows the kinked small filament (07:00:15 UT) associated with the brightenings near its southern leg. The twisted (writhed) fields at the apex of the filament is much clearer here. The southern leg of the filament detached from the surface as a result of magnetic reconnection and showed an unwinding motion of the apex and northern leg in the counterclockwise direction (Figs. 3e, f). The apex of the filament also detached and showed a failed eruption similar to the AIA $304 \AA$ images. Most of the plasma is drained back to the solar surface. These images clearly demonstrate the dynamical evolution of the filament associated with the kink instability.

\subsection{Hard $X$-ray emission}

To investigate the particle acceleration sites during the flare associated with the kinked small filament eruption, we used hard X-ray images from the Reuven Ramaty High Energy Solar Spectroscopic Imager (RHESSI; Lin et al. 2002). We used the PIXON algorithm technique to reconstruct the RHESSI images because of its most accurate image photometry (Metcalf et al. 1996; Aschwanden et al. 2004). We adopted an integration time of $20 \mathrm{~s}$ for the image reconstruction in the $6-12 \mathrm{keV}$ and $12-25 \mathrm{keV}$ energy channels. The hard X-ray emission was limited to these energy channels and was not detected in higher energy bands.

Figure 4 displays the selected AIA 1600, 1700, and $131 \AA$ images overlaid by RHESSI hard X-ray images contours in the 6-12 keV (red) and 12-25 keV (blue). We used the AIA 1600 and $1700 \AA$ images to explore the photospheric brightenings associated with accelerated particles from the corona (i.e., hard X-ray emission). Flare-heated loops and associated (hot and cool) magnetic structures $(0.4,11$, and $16 \mathrm{MK})$ are clearly visible in the AIA $131 \AA$ channel. The co-temporal AIA images overlaid with the RHESSI image contours are shown in the first and second rows. A small filament (indicated by arrow, Fig. 4a') was observed before the flare onset, and no brightening was observed in AIA $1600 \AA$ at 06:57:05 UT. The filament activation and subsequent rising motion was closely associated with the formation of a hot loop (Figs. $4 \mathrm{~b}$ and $\mathrm{b}^{\prime}$ ) near the southern leg of the filament. A hard X-ray source (observed in both energy bands, marked by A) was found to be cospatial with the hot loop. Furthermore, we see a clear loop structure in $12-25 \mathrm{keV}$ channel that shows the two footpoints (FP1 and FP2) and a looptop source (LT) during 06:58:40-06:59:00 UT. As the filament rises, an another source B is formed (Figs. 4d and $d^{\prime}$ ), which is located above the filament apex. The underlying flare loop becomes brighter as the filament moves up because of the progressive magnetic reconnection associated with the breaking of the southern leg. Source $\mathrm{B}$ in $6-12 \mathrm{keV}$ has an elongated structure parallel to the flare ribbons. Simultaneously, we observe the brightening along the northern side of the filament (two kernals K1 and K2 are marked by arrows, Fig. 4d). The height of source B increases with the rising motion of the filament (Figs. $4 \mathrm{e}, \mathrm{e}^{\prime}, \mathrm{f}, \mathrm{f}^{\prime}$ ). Furthermore, the structure of source B becomes elongated during 07:01:2007:01:40 UT (Figs. 4g and $\mathrm{g}^{\prime}$ ). Later, two sources C and D emerge along the sides of the filament apex (Figs. $4 \mathrm{~h}$ and $\mathrm{h}^{\prime}$ ).

The appearance of source $A$ at the beginning of the flare suggests the occurrence of magnetic reconnection above or near the southern leg of the filament, which causes the formation of a hot underlying loop, observed in AIA $131 \AA$. Consequently, the filament moved up and the southern leg broke apart. Furthermore, source B above the filament apex indicates particle acceleration (energy release site) probably caused by the magnetic reconnection above the filament, resulting in the appearance of an overlying small flux rope like structure (which we discuss in the next section). In addition, the elongation of source $\mathrm{B}$ and the appearance of sources $\mathrm{C}$ and $\mathrm{D}$ confirm the particle acceleration from the filament apex in the north and south directions.

Using the RHESSI and TRACE observations, Ji et al. (2003) have reported a hard X-ray source $(12-25 \mathrm{keV})$ above a largescale filament in the beginning of the flare. Later, the filament 

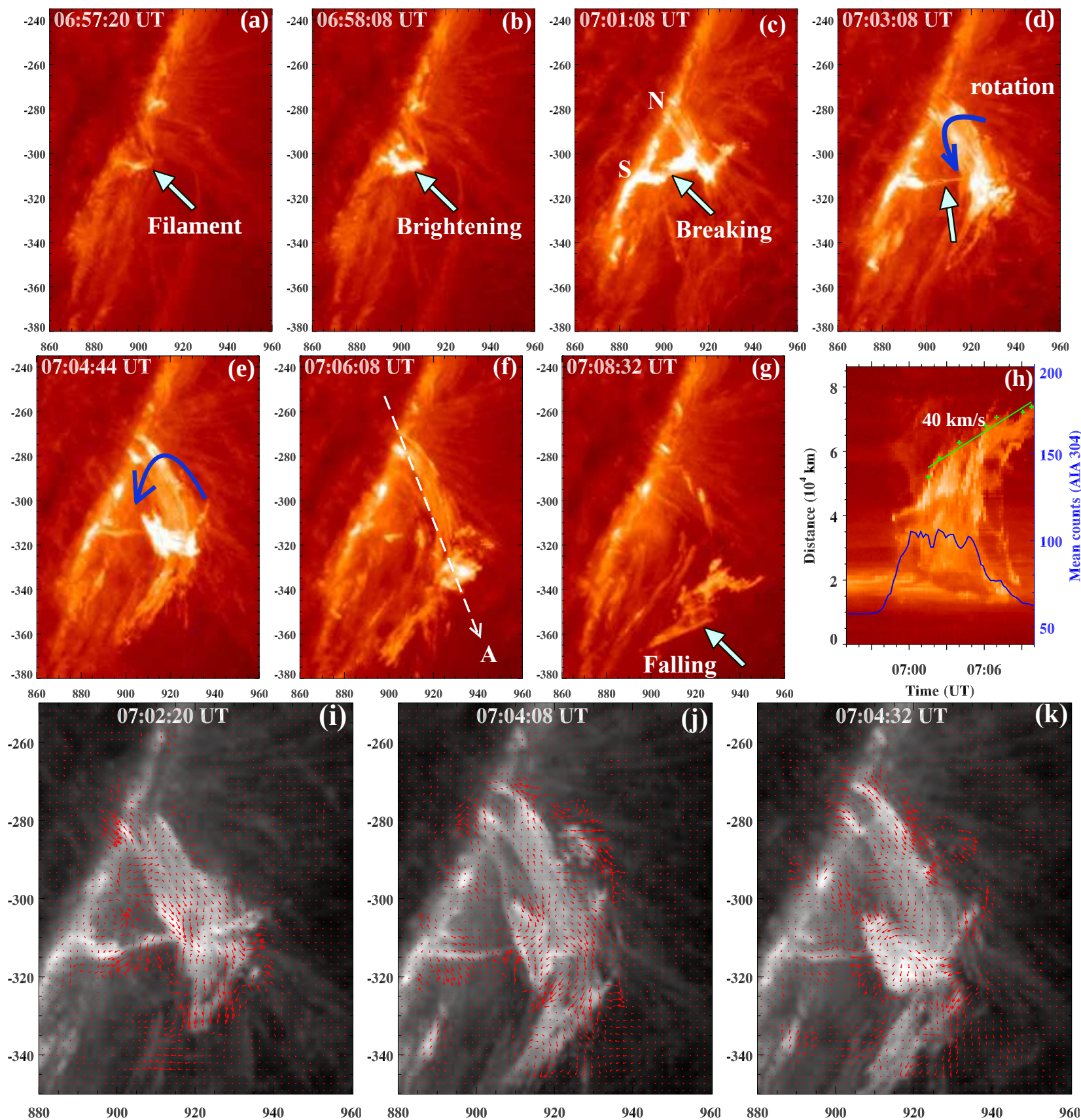

Fig. 2. a)-g) SDO/AIA EUV images in the $304 \AA$ channel $(T \sim 0.05 \mathrm{MK})$ showing the eruption of a kinked small filament associated with the C3.9 flare on 30 April 2012. N and S represent the northern and southern legs of the filament. The blue arrows show the unwinding motion of the northern leg and the top portion of the filament. $X$ and $Y$ axes are in arcsecs. h) Space-time plot along the slice A (shown by the dotted line in panel f)) plotted with AIA $304 \AA$ mean counts in blue curve. i)-k) Apparent velocity field computed with DAVE using AIA $304 \AA$ images, showing the plasma flows in and around the prominence threads. The longest arrow (in the panels) corresponds to a speed of i) $204 \mathrm{~km} \mathrm{~s} \mathrm{~s}^{-1}, \mathbf{j}$ ) $178 \mathrm{~km} \mathrm{~s}^{-1}$, and $\left.\mathbf{k}\right) 158 \mathrm{~km} \mathrm{~s}^{-1}$. The temporal evolution of the kinked filament in $304 \AA$ can be found in the online movie.

was associated with the kink instability and failed to erupt. The authors suggested that the reconnection (i.e., energy release) must have occurred above the filament. This result is not supported by the standard flare model (i.e., CSHKP), because reconnection takes place below the filament or flux rope in the CSHKP model. Alexander et al. (2006) have also studied the same event and confirmed the presence of a hard X-ray source $(12-25 \mathrm{keV})$ in the corona above the filament before the main activation phase. They identified a second coronal hard X-ray source under the apex of the strongly kinked filament during the eruption. They interpreted this as the energy release site at the vertical current sheet formed in between the crossing legs of the filament, as suggested in numerical simulations of kink instability (Török et al. 2004). We also observed the hard X-ray source above the filament similar to these observational reports, but no hard X-ray source in between the crossing legs of the filament.

\subsection{Flux rope formation and eruption}

We used the AIA $94 \AA$ base difference and AIA $131 \AA$ runningdifference images (Fig. 5) to investigate the hot plasma structures 

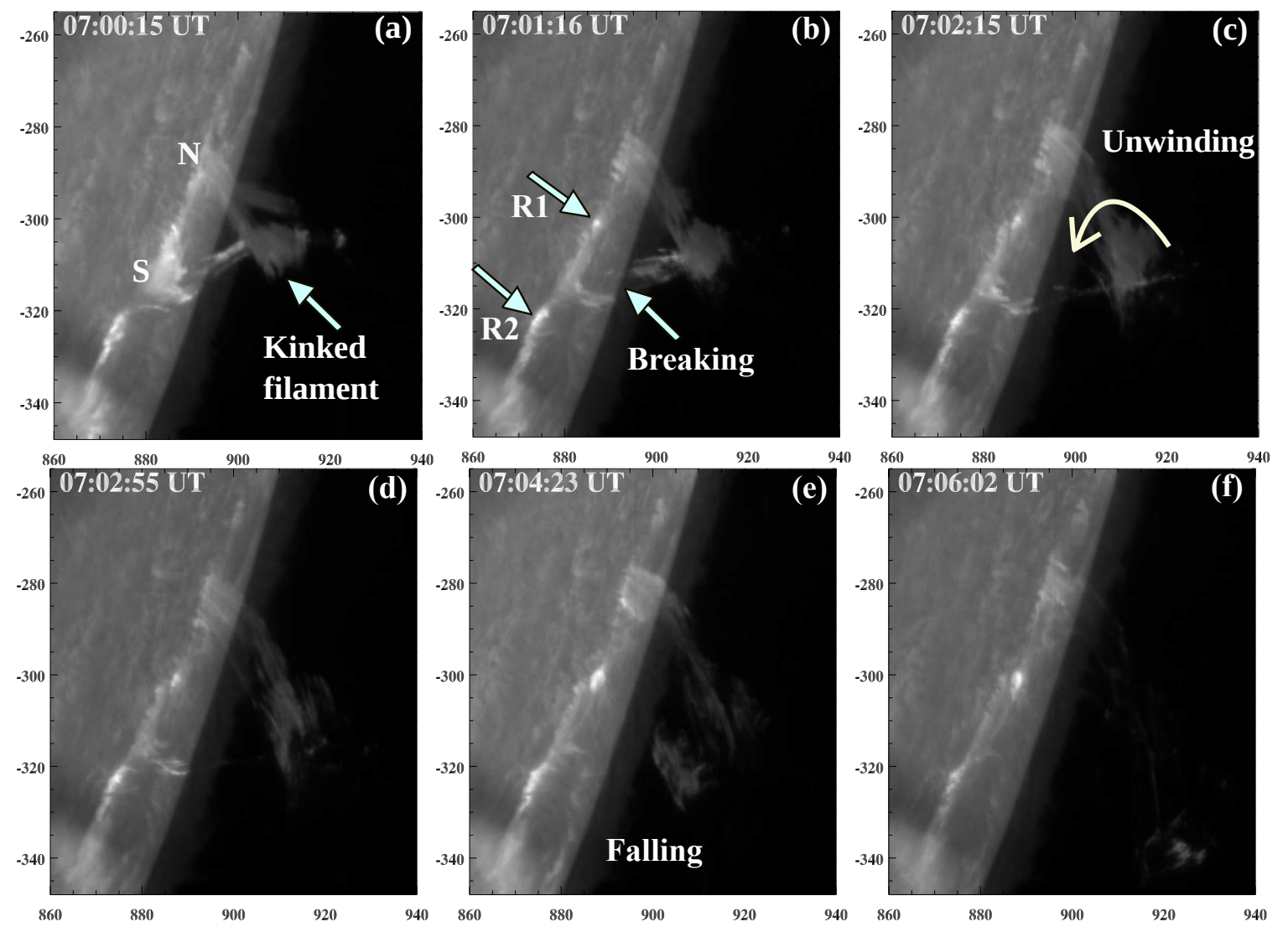

Fig. 3. Hinode/SOT $\mathrm{H} \alpha$ images showing the eruption of the kinked small filament associated with the C3.9 flare. R1 and R2 indicate the flare kernels.

associated with the kinked filament and the C3.9 flare. We chose a time difference of one minute to construct these images. The flux rope was observed only in these two hot channels, which suggests a high temperature of the flux rope (i.e., 6.3-16 MK) (Cheng et al. 2012; Hannah \& Kontar 2013). Figures 5a,b show the activation of the small filament along with the formation of a underlying flare loop near its southern leg during 6:56-6:57 UT. As the filament reached the height of about $18 \mathrm{Mm}$, we notice the first appearance of a very small flux rope structure (red dotted curve) above the filament during magnetic reconnection at 06:58 UT.

The underlying hot loop near the southern leg of the filament is shown by the blue dotted curve. Furthermore, Figures $5 \mathrm{e}-\mathrm{h}$ display the dynamics of the flux rope (above the filament) during the slow rise of the filament. We observe the appearance of a sheared loop \#1 at 06:59:21 UT (Fig. 5f) during the reconnection process above the filament, and an another loop \#2 at 07:00:09 UT. The signatures of particle acceleration along these loops are observed as footpoint brightenings in the simultaneous AIA 1700 image (Fig. 4d). In addition, the appearance of loop \#3 at 07:00:57 UT also indicates reconnection above the filament. Simultaneously, we observed the lateral expansion of the flux rope along the southward direction. The process of the flux rope's slow rise and expansion continued for $\sim 15-20 \mathrm{~min}$ (Fig. 5i-k). The bottom panels show the larger field of view, and the size of the upper panels is marked by the blue dotted rectangular box (Fig. 5i). The twisted flux rope structure was best observed in AIA $131 \AA$ during 7:13-7:15 UT. The flare loop formation below the flux rope is evident at 07:15:09 UT (Fig. 5k). The large-scale flux rope expansion and formation of the underlying loops are illustrated by dotted curves (Fig. 51).
We used soft X-ray images of the active region recorded by the Hinode X-ray Telescope (XRT, Golub et al. 2007). The field of view of each image is $384 \times 384$ pixels with a resolution of 1.02 arcsec pixel $^{-1}$. The images are captured by a Ti-poly filter containing a broad temperature response with a flat peak at $10^{6.9} \mathrm{~K}$. Figure 6 displays the selected images of the eruption site shortly before and during the flux rope eruption. These images are coaligned with the AIA images. Figure 6a shows the preexisting arcade loop systems in the active region at 06:54:46 UT. We see the formation of a bright underlying loop (marked by the arrow in Fig. 6b) with the activation of a small filament similar to the AIA $94 \AA$ images. The flux rope structure appeared above the kinked filament (yellow dotted curve), and the underlying flare loop formed at the southern leg of the filament at 07:00:07 UT (Fig. 6c). The flux rope structure is much clearer in these images than in the AIA images. It looks like a small-scale flux rope appeared above the kinked filament during magnetic reconnection. We include a simultaneous $131 \AA$ image (Fig. 6d) to compare the structure in X-ray and EUV. The structure observed above the kinked filament in $131 \AA$ is consistent with the X-ray structure. Note that both images (EUV and X-ray) are sensitive to $\sim 10 \mathrm{MK}$ plasma temperature. This structure slowly expands and probably reconnects with the ambient fields within next 10-12 min, thus forming a large-scale structure. The bottom row shows the evolution of the flux rope in the low corona in a larger field of view than the upper row. Figures 6e and f show a clear twisted flux rope expanding laterally in the southward direction.

Initially, at about 07:00 UT, we cannot rule out that the structure (above the filament) is an arcade of loops, or a very small flux rope. However, this structure was later linked to a large flux 

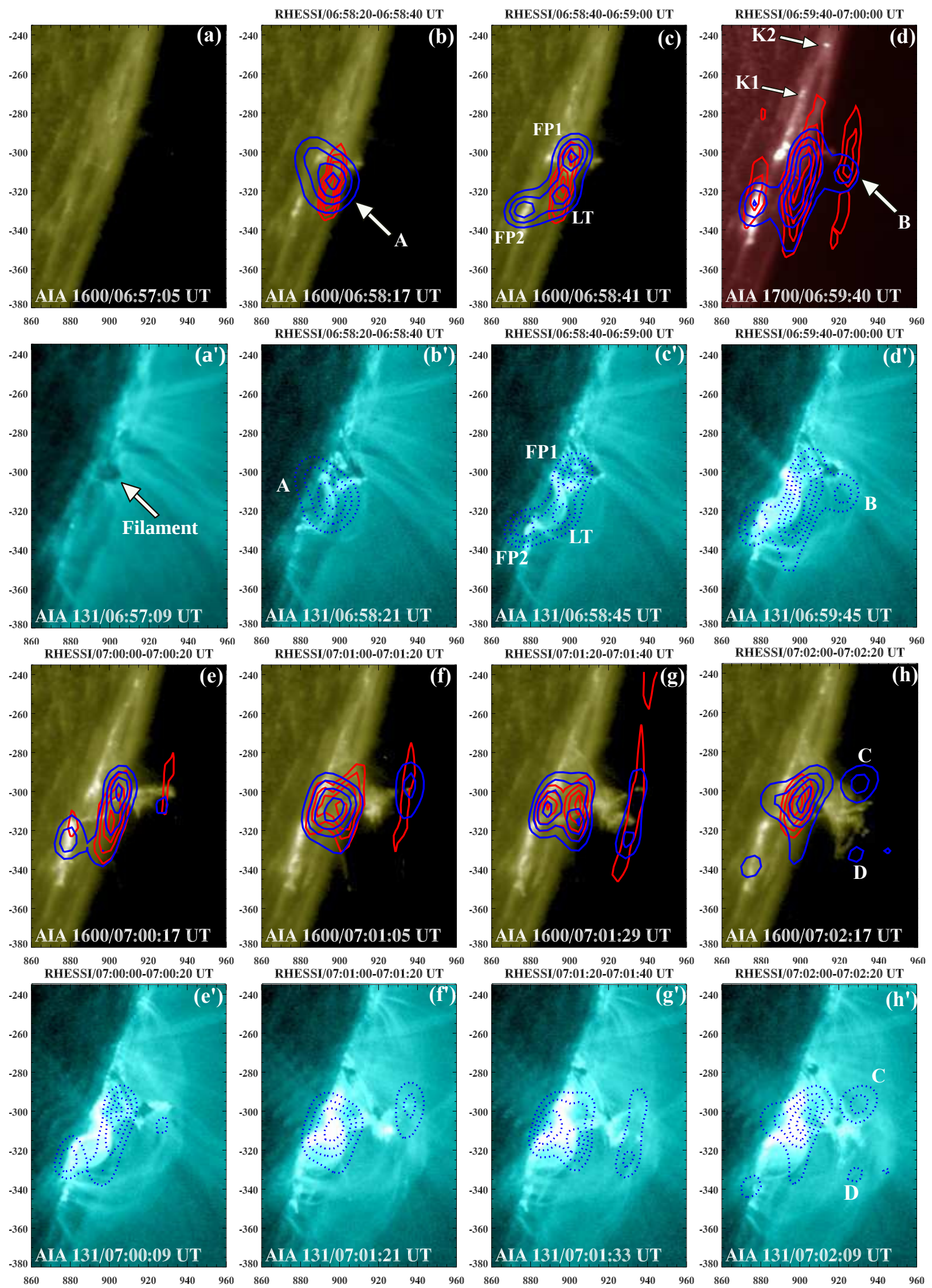

Fig. 4. SDO/AIA 1600, 1700, and $131 \AA$ images overlaid with the RHESSI hard X-ray image contours in the 6-12 keV (red) and 12-25 keV (blue) energy channels. The contour levels are $30 \%, 50 \%, 70 \%$, and $90 \%$ of the peak intensity. 
P. Kumar and K.-S. Cho: Multiwavelength observation of a large-scale flux rope eruption above a kinked small filament

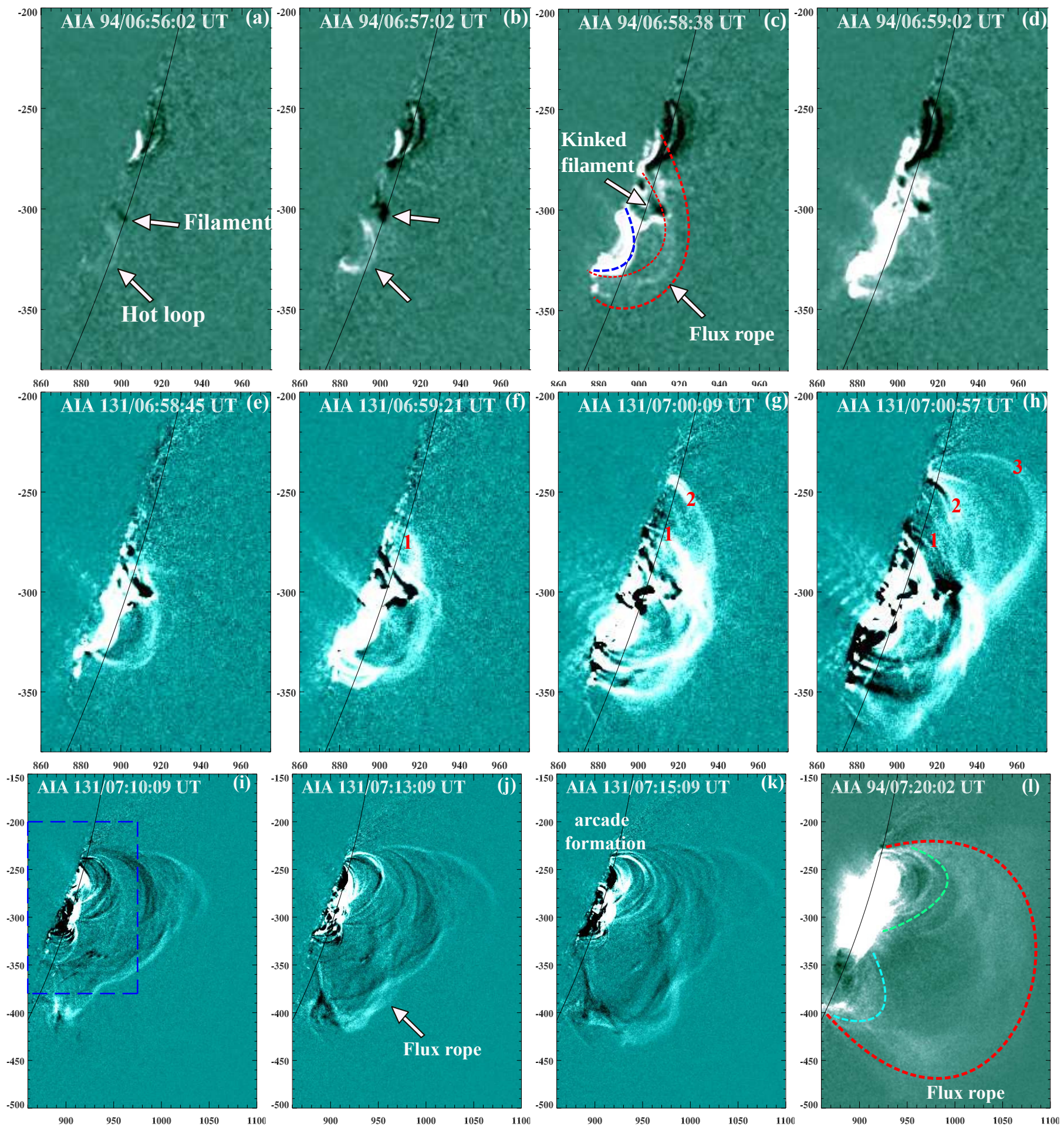

Fig. 5. SDO/AIA $94 \AA$ base difference and $131 \AA$ running-difference images showing the formation and ejection of the twisted flux rope above the kinked filament during magnetic reconnection. The red dotted curve shows the flux rope, the other curves indicate the underlying closed loops (panels c) and l)). The blue rectangle (panel i)) represents the size of the upper panels.

rope structure (observed in X-ray and EUV) after a series of magnetic reconnection above the small filament (within the arcade loops). Therefore, it is also possible that the initial structure above the filament is a part of the flux rope observed later during magnetic reconnection. The observed structure (after 07:10 UT) most likely is a magnetic flux rope because (i) it is observed only in the AIA hot channels (AIA 131 and $94 \AA$ ), whose morphology and temperature $(T \sim 8 \mathrm{MK})$ are quite similar to the flux ropes reported in recent case studies (Zhang et al. 2012; Patsourakos et al. 2013; Cheng et al. 2014). (ii) The structure is aligned and appeared above the neutral line (along the flare ribbons observed in the AIA $1600 \AA$ ) and the orientation of the structure is across the post flare arcade (cusp) loops formed after the eruption (see Fig. 9d). Furthermore, Fig. 6h displays the AIA $171 \AA$ reversecolor image (at 09:15 UT) during the decay phase of the flare (after the flux rope eruption). Generally, the orientation of the 

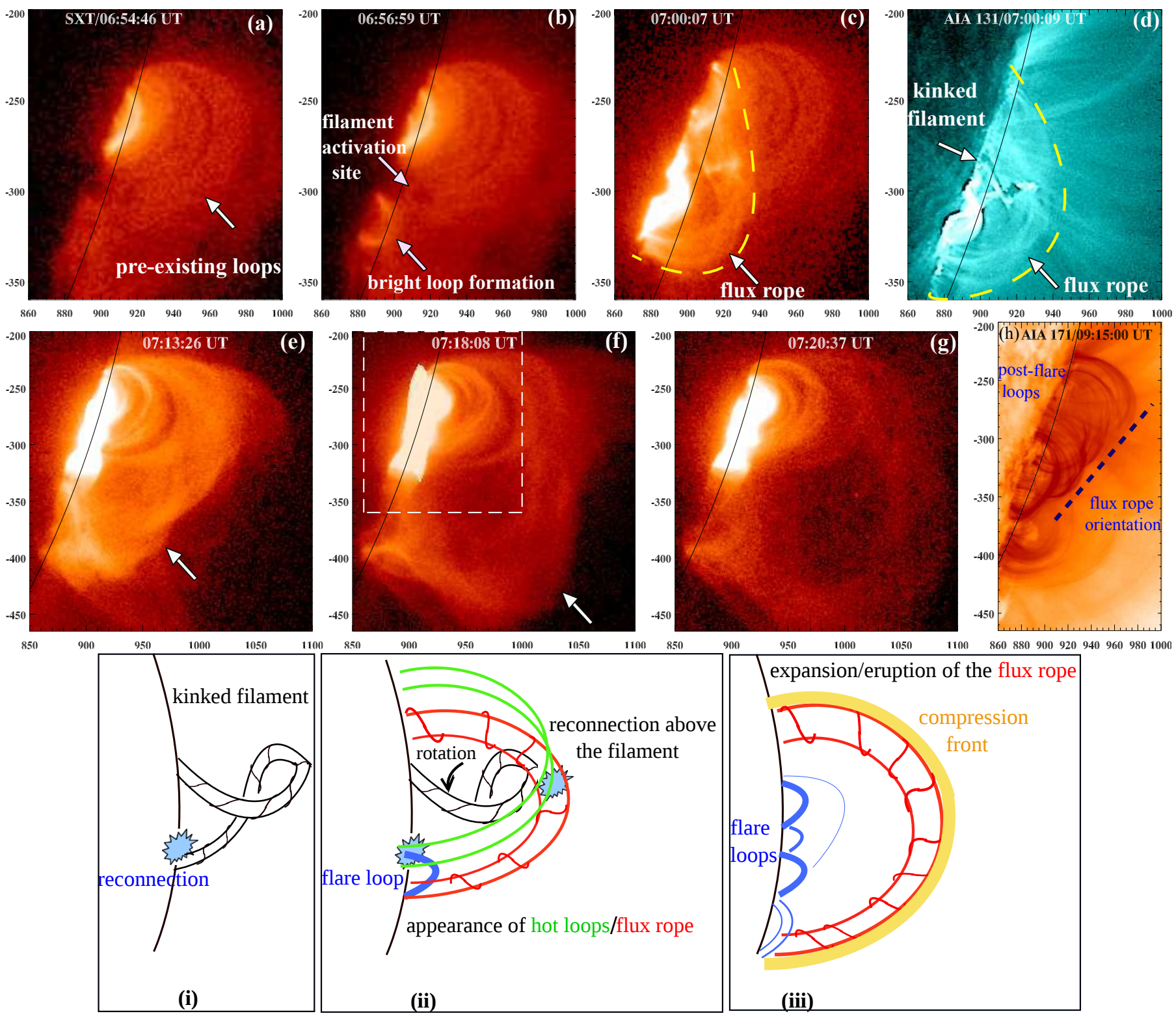

Fig. 6. Hinode/SXT images of the eruption site showing the evolution of the flux rope during the flare. The appearance of the flux rope is shown by a yellow dotted curve c) and d). The dotted rectangular box in panel f) represents the field of view of the upper row panels. Panel g) shows the post-flare loops observed in the AIA $171 \AA$ channel, and the orientation of the flux rope is marked by the dotted lines. Bottom: schematic cartoon showing the different stages of the eruption.

flux rope can be derived from the post-flare loops (Yurchyshyn 2008). The flux rope lies along the neutral line (marked by the dotted line), which agrees with the flux rope elongation observed in the soft X-ray images.

On the basis of our observational findings, we draw a schematic cartoon to explain the different stages of the eruption. The bottom panel of Fig. 6 shows (i) reconnection at the southern footpoint of the filament and its subsequent counterclockwise motion; and (ii) the breaking of the southern leg and formation of a hot underlying flare loop (blue). Reconnection above the filament results in the formation of hot loops and the appearance of the flux rope structure; (iii) the lateral and radial expansion of the flux rope leads to the formation of a compression front around it (observed in the $171 \AA$ channel). The counterclockwise rotation of the filament reveals the left-handed twist.

To study the morphology the flux rope in different AIA channels, we display the co-temporal AIA running difference images in $131,94,335$, and $171 \AA$ channels at 07:19 UT (Fig. 7). The AIA $131 \AA$ image shows a clearly twisted structure. The flux rope was not initially visible in $335 \AA$ during its formation process, but later some part of the flux rope (north direction) was observed during its acceleration phase (Fig. 7c). The expansion of the flux rope results in the formation of a cool compression front surrounding the flux rope (green contours), which was observed in the AIA $171 \AA$ channel (Fig. 7d). The temporal evolution of the flux rope in four AIA channels is shown in the animation attached to Fig. 7.

For the kinematics of the flux rope, we used slice cuts S1, S2, S3, and S4 in the AIA 131, 94, 335, and $171 \AA$ channels, respectively. The stack plot is shown in Fig. 8a. Slices S1 and S2 show the slow rising phase of the flux rope at $\sim 07: 00$ UT and an impulsive acceleration at $\sim 07: 14$ UT. Slice S3 shows only the acceleration phase of the flux rope in $335 \AA$. The location of slice S3 is different from S1 and S2. It is likely that the initial 
P. Kumar and K.-S. Cho: Multiwavelength observation of a large-scale flux rope eruption above a kinked small filament
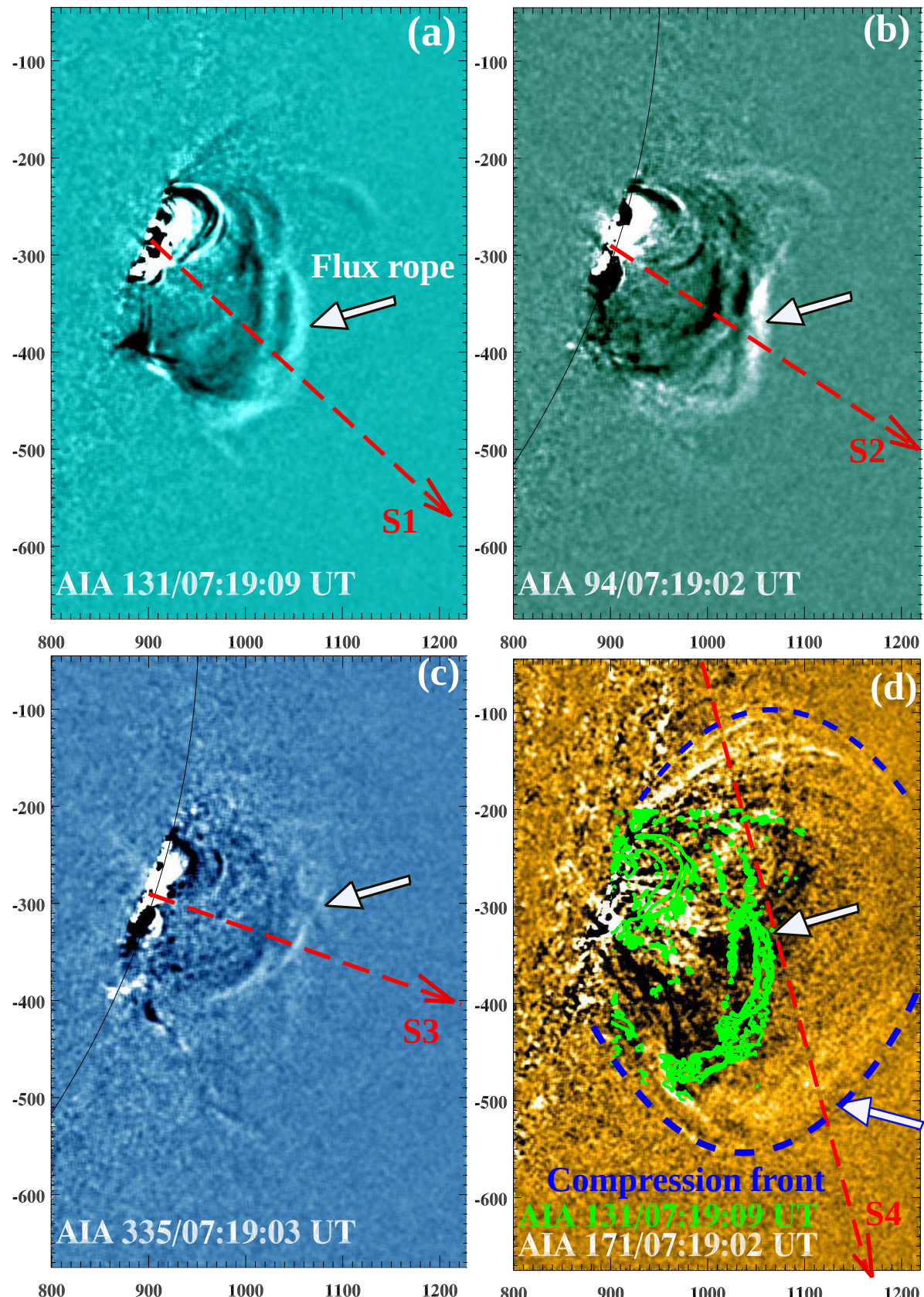

Fig. 7. SDO/AIA running difference images in the 131, 94, 335, and $171 \AA$ channels showing the flux rope eruption. S1, S2, S3, and S4 are the slice cuts used for the stack plots. The flux rope contours (green) of AIA $131 \AA$ are overlaid on the AIA $171 \AA$ image (panel d)). The blue dotted curve in AIA $171 \AA$ indicates the cool compression front of the CME. The temporal evolution of the flux rope and the associated flare in 131, 94, 335 , and $171 \AA$ can be found in the online movie.

temperature of the flux rope is high enough to be visible only in the 131 and $94 \AA$ channels as it is appeared during the magnetic reconnection. Later, it might cool down to a temperature of about $2.5 \mathrm{MK}$ so that part of it was observed in $335 \AA$ during the acceleration phase. This indicates multitemperature plasma within the flux rope. Alternatively, the reconnection of the flux rope with the preexisting field lines may produce some portion of the flux rope to be visible in $335 \AA$. The lateral expansion of the CME was observed in AIA $171 \AA$, which shows a cool compression front around the flux rope. From the linear fit, the estimated lateral speed of the CME was $\sim 50 \mathrm{~km} \mathrm{~s}^{-1}$ (north) and $\sim 128 \mathrm{~km} \mathrm{~s}^{-1}$ (south). We also note EUV disturbances or brightenings (at 07:02 and 07:14 UT) and contraction or closing of the $171 \AA$ loop (at 07:28 UT) in the $171 \AA$ stack plot. These may be additional evidence for magnetic reconnection above the filament and below the newly formed flux rope.

The top panel of Fig. 8b displays a height-time plot of the flux rope tracked in the stack plot of slice S2 (AIA $94 \AA$ ). We used a cubic spline-smoothing scheme to reduce the fluctuations in data points (marked by +; Vršnak et al. 2007). We assumed an error of four pixels $\left(2.4^{\prime \prime}\right)$ in the height estimation. The middle panel shows the speed profile of the flux rope, which is computed from the height-time measurements using a numerical differentiation with three-point Lagrangian interpolation (Zhang et al. 2004). The bottom panel displays the acceleration profile and the evolution of the hard X-ray flux in $12-25 \mathrm{keV}$ 

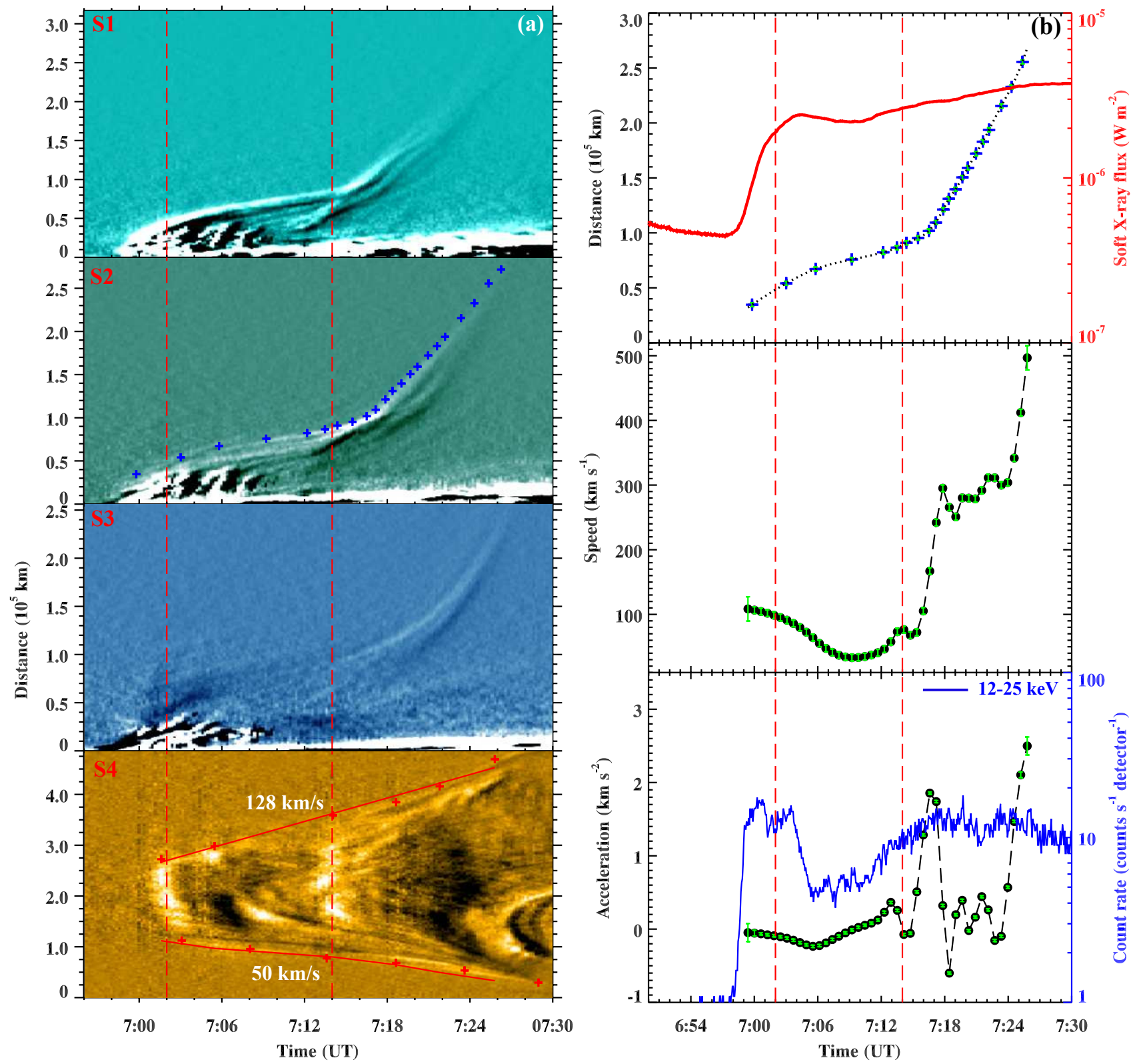

Fig. 8. a) Space-time plots along slices S1, S2, S3, and S4 using the AIA 131, 94, 335, and $171 \AA$ images. b) Top: height-time profile of the flux rope and GOES soft X-ray flux (red) in the 1-8 $\AA$ channel. Middle: speed profile derived from the height-time measurements of the flux rope. Bottom: acceleration profile of the flux rope plotted with RHESSI hard X-ray flux (blue) in the $12-25 \mathrm{keV}$ energy channel. The two vertical dotted lines indicate the timing of the $171 \AA$ brightenings and hard X-ray bursts.

(blue). The uncertainty in the speed and acceleration is mainly due to the error in the height measurement. The initial speed of the flux rope was $\sim 100 \mathrm{~km} \mathrm{~s}^{-1}$ during the slow rising process at about 07:00 UT. It reduced to about $30-40 \mathrm{~km} \mathrm{~s}^{-1}$ during 7:02-07:10 UT, and again started to increase at $\sim 7: 12 \mathrm{UT}$. We note an impulsive increase in the speed up to $\sim 300 \mathrm{~km} \mathrm{~s}^{-1}$ at $\sim 7: 16$ UT. During the whole process, the flux rope speed varies from 40 to $500 \mathrm{~km} \mathrm{~s}^{-1}$. The flux rope does not show significant acceleration during the first hard X-ray burst at about 07:00 UT. Furthermore, we note an acceleration of $\sim 2 \mathrm{~km} \mathrm{~s}^{-2}$ at 7:16 UT, which coincides well with the rise in the hard X-ray flux during 07:08-07:24 UT.

Solar TErrestrial RElations Observatory (STEREO) SECCHI-A (ahead; Wuelser et al. 2004; Howard et al. 2008) observed the same AR near the disk center. We used EUVIA $284 \AA$ images to see the magnetic field topolgy of the active region. The size of the image is $2048 \times 2048$ pixels $\left(\sim 1.6 \operatorname{arcsec}_{\text {pixel }}{ }^{-1}\right)$, covering a field of view out to $1.7 R_{\odot}$. The peak temperature response for the $284 \AA$ channel is $2.2 \mathrm{MK}$ (Aschwanden et al. 2008). Fortunately, we found two images of the eruption site, before and after the flux rope eruption. Figure 9 shows the simultaneous images in EUVI-A $284 \AA$ and AIA $94 \AA$ channels at 06:16 and 08:16 UT. Figure 9a shows a reverse $J$ shaped structure in the AR. We used the routine scc_measure to compare the magnetic structures of the AR in 284 and $94 \AA$. The approximate positions of the chosen structures are marked by + symbols with different colors in both images. The location of the kinked filament is overplotted in Fig. 9b. The comparison of images indicates that the filament was located at the magnetic separatrix and below the reverse J-shaped loop. Figure 9c shows the post-eruptive arcade system formed at the site of filament activation and the J-shaped loop. The cusp-shaped loops were 
P. Kumar and K.-S. Cho: Multiwavelength observation of a large-scale flux rope eruption above a kinked small filament
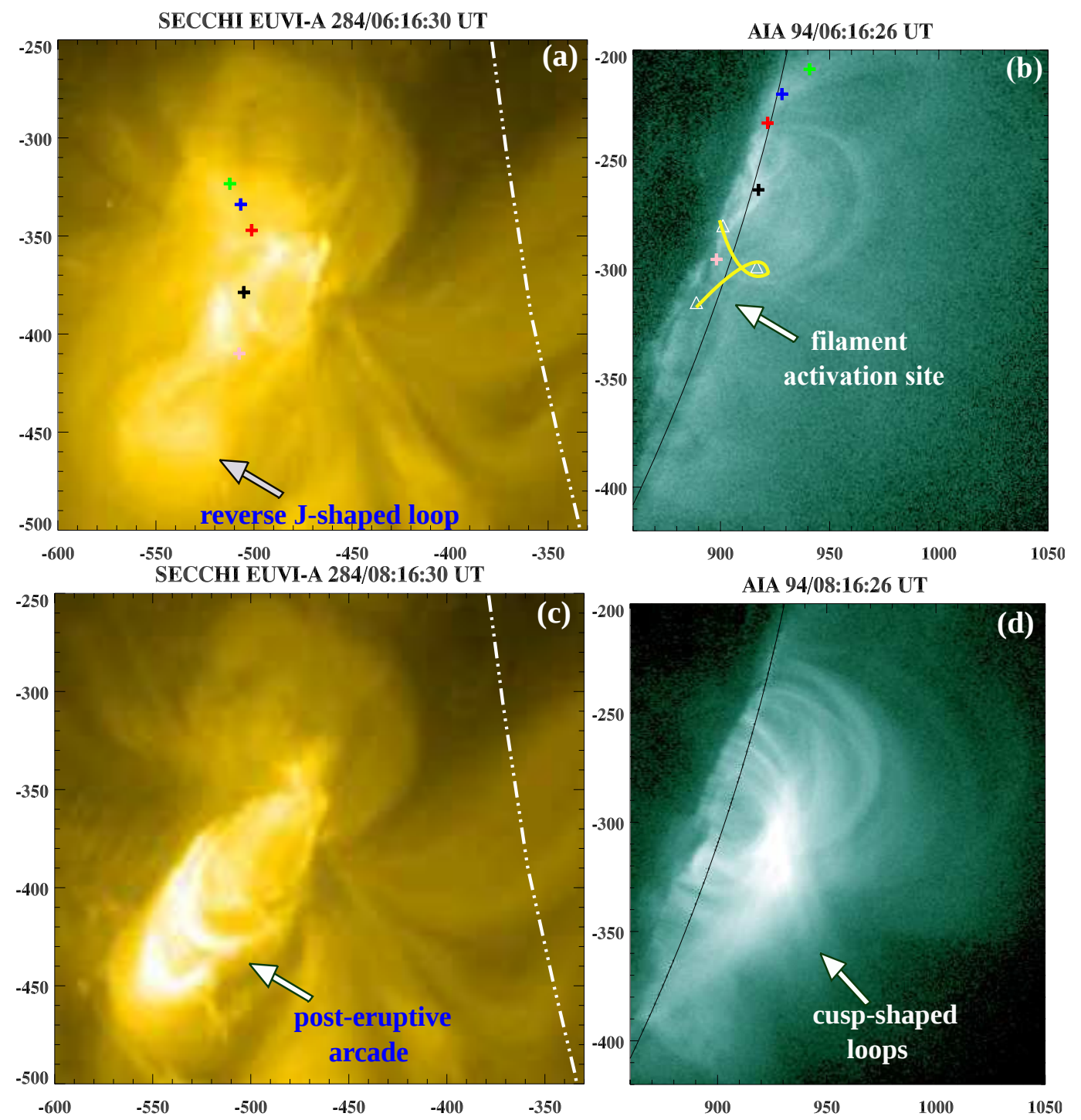

Fig. 9. STEREO SECCHI EUVI-A images showing the magnetic field configuration of the active region in the $284 \AA$ channel before a) and during $\mathbf{c}$ ) the flare. For comparison, the approximate positions of the magnetic structures are marked by + . The position of the filament activation is drawn by using AIA $131 \AA$ image at 07:00 UT. The limb position from the AIA image is overplotted on SECCHI images (white dotted lines). AIA $94 \AA$ images (b) and d)) of the eruption site.

observed in AIA 94 (and 131) ^ images (Fig. 9d). We also observed the supra-arcade downflows (e.g., Innes et al. 2014) above the cusp-shaped loops, which is indirect evidence of magnetic reconnection below the flux rope during the eruption. The combined magnetic field configuration (i.e., northern loops from SXT/AIA $94 \AA$ and southern sheared field lines from SECCHI $284 \AA$ ) from two-point observations also indicates quasi-separatrix layers (QSLs; Demoulin et al. 1996, 1997), where the magnetic reconnection takes place above the kinked filament and cusp loops are formed during the decay phase of the flare.

\subsection{EUV disturbances and brightenings}

To see the coronal responses of the magnetic reconnection during the flux rope appearance, we generated AIA $171 \AA$ running-difference images. Figure 10 displays some of the selected images during the flare. We observed multiple propagating brightenings in the corona associated with the flux rope formation during the magnetic reconnection. The first EUV brightening or pulse was observed during the first hard X-ray burst at about 07:00 UT (Figs. 10a and b). The brightening occurred above the small filament, which closely matched the coronal hard X-ray sources (sources B, C, and D in Fig. 4). The second brightening was observed during the second hard X-ray burst at about 07:12 UT close to the flux rope (Fig. 10d). More details about the spatial location and evolution of the EUV waves can be found in the AIA four-channel composite movie (attached to Fig. 7). The timing and location of these short brightenings suggest that they are closely associated with the magnetic reconnection and may be interpreted as multiple EUV wavefronts (Liu et al. 2011). The formation of southward closed loop systems (marked by the dotted curve) result from magnetic reconnection (Fig. 10c). These underlying closed loops are also observed in the AIA $94 \AA$ channel after the second hard X-ray burst (Fig. 51).

\section{5. $C M E$}

To see the evolution of the CME in a larger field of view than that of AIA, we used the SWAP (Sun Watcher using Active 

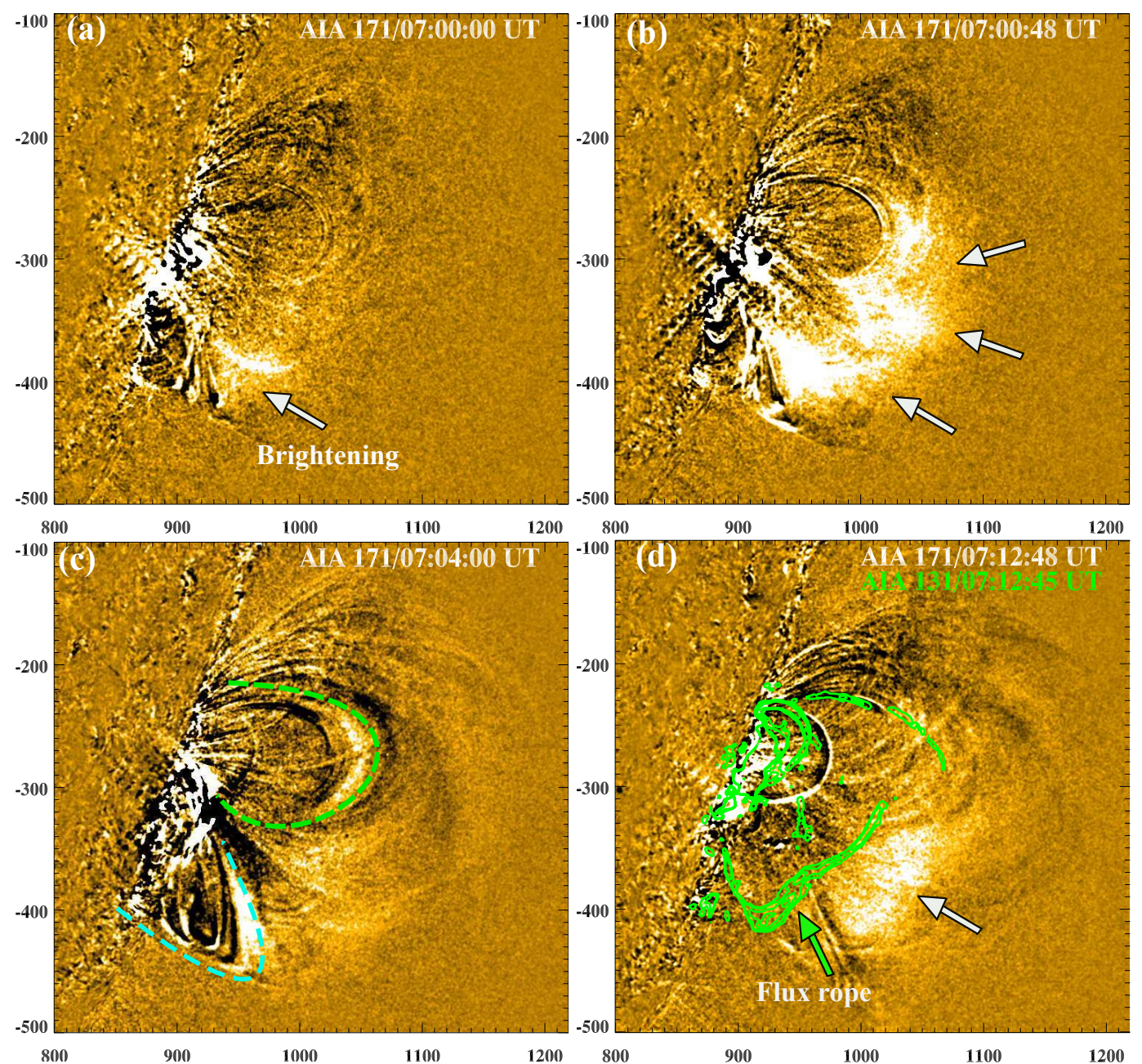

Fig. 10. a)-c) AIA $171 \AA$ running-difference images showing the transient EUV brightenings (marked by arrows) above the flare site. The two underlying loop systems are marked by the dotted curves. d) $171 \AA$ running-difference image overlaid by $131 \AA$ running-difference image contours (green).

Pixel System Detector and Image Processing) $174 \AA$ running difference image (7:25:10-07:23:00 UT) shown in Fig. 11a. The SWAP telescope is a compact EUV imager onboard the PROBA2 (PRoject for Onboard Autonomy) micro-satellite, which provides coronal images at a temperature of $\sim 0.8 \mathrm{MK}$ (Berghmans et al. 2006; Halain et al. 2010). The CME frontal loop shown by blue curve is clearly observed, which agrees well with the AIA $171 \AA$ images of the low corona (Fig. 7d). Note that we did not observe the flux rope in cool channels. Two sets of underlying loops are shown by dotted curves that indicate the reorganization of coronal magnetic field by magnetic reconnection.

The white-light running-difference images from SOHO/LASCO C2 (Brueckner et al. 1995; Yashiro et al. 2004) reveal the flux rope structure behind the CME frontal loop, which is shown by arrows in Figs. 11b and c. It seems to cover the cavity region of the CME. The dark cavities (surrounded by bright core, i.e., prominence) are often observed in the three-part structure of CMEs, and are explained by the flux rope model (Illing \& Hundhausen 1985, 1986; Bạk-Stẹślicka et al. 2013). In our case, the filament failed to erupt, and the cavity part is covered by the flux rope.

The top panel of Fig. 12 shows the composite height-time profile of the flux rope (observed in AIA $94 \AA$ ) and the frontal loop from LASCO C2 and C3. The speed of the flux rope is derived from the three-point Lagrangian interpolation method (see Fig. 8). The speed of the CME frontal loop is estimated from a second-order polynomial function fitted to the heighttime measurements. The average speed of the CME in the LASCO field of view is $\sim 992 \mathrm{~km} \mathrm{~s}^{-1}$, and the acceleration is $\sim-4.98 \mathrm{~m} \mathrm{~s}^{-2}$. The bottom panel displays the dynamic radio spectrum in $25-180 \mathrm{MHz}$ observed from Learmonth radio observatory, Australia. A type II radio burst (25-102 MHz) was observed during the impulsive acceleration of the flux rope between 07:27 UT-07:33 UT. The timing and formation height of the type II source indicates the piston driven shock ahead of the CME front. From the frequency drift rate, the speed of the type II excitor was $\sim 645 \mathrm{~km} \mathrm{~s}^{-1}$, which should be close to the flux rope speed at the formation height of type II burst. Interestingly, no type III burst was observed during the activation of the kinked filament at 07:00 UT. This suggests that there is no opening of the field lines into the interplanetary medium. It is also possible that the flux rope above the small filament did not allow the particles to escape outward. The first impulsive rise in SXR flux ( 07:00 UT) is not synchronized with the CME speed (i.e., impulsive acceleration), as shown by Zhang \& Dere (2006). As discussed in the first section, this is related to the magnetic reconnection at the filament leg and apex. However, the impulsive acceleration of the flux rope from $\sim 07: 12$ UT onward 
P. Kumar and K.-S. Cho: Multiwavelength observation of a large-scale flux rope eruption above a kinked small filament
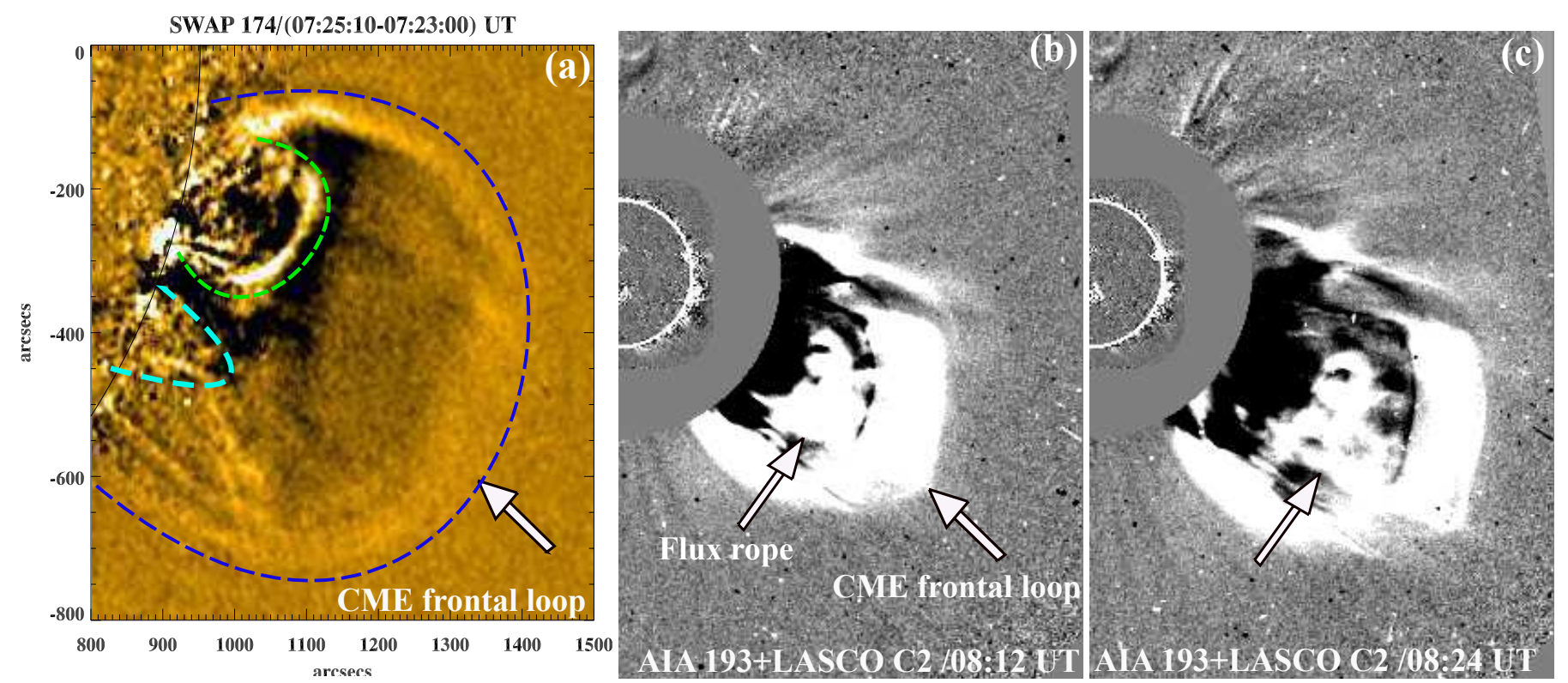

Fig. 11. a) SWAP $174 \AA$ running-difference image showing the compression front of the CME (blue dotted curve) and underlying loop systems (dotted curves). b)-c) AIA $193 \AA$ and LASCO C2 composite running-difference images. The flux rope and the CME frontal loop are shown by arrows.

is synchronized with the gradual rise of the SXR flux, which might be related to the successive magnetic reconnection below the flux rope. In addition, the tether-cutting reconnection below the flux rope usually increases the magnetic pressure by adding the poloidal flux that enhances the hoop force of the flux rope (Vršnak et al. 2004; Zhang \& Dere 2006).

\section{Summary and discussion}

We presented a multiwavelength study of a flux rope eruption associated with a failed eruption of the kinked small filament and the C3.9 flare. The main results of this study are summarized as follows:

(1) The activation and slow rising motion $\left(\sim 40 \mathrm{~km} \mathrm{~s}^{-1}\right)$ of the kinked small filament was associated with the formation of a hot loop near its southern leg. The first magnetic reconnection possibly occurred at or above the southern leg of the filament that causes the formation of a hot loop and the breaking of the southern leg. RHESSI hard X-ray images (12-25 keV) clearly show the two footpoints and a looptop source coinciding with the underlying hot loop.

After breaking the leg, the filament twist is converted into the writhe, as seen clearly in the SOT H $\alpha$ images. The filament showed unwinding motion of the apex and northern leg in counterclockwise direction (left-handed twist) and fell back to the solar surface within $\sim 10 \mathrm{~min}$. The morphology of the filament and its rotation support the occurrence of a kink instability and the relaxation of the twist during the eruption (Török \& Kliem 2005; Kliem et al. 2012).

(2) Before the flare onset, we observed a small filament in the AIA $304 \AA$ channel. The flux rope appeared during the second magnetic reconnection above the apex of the small filament. We have sufficient evidence to support this argument: (i) appearance of the RHESSI hard X-ray source (12-25 keV, source B) above the filament apex during 06:59:40-07:00 UT, suggesting that the particle acceleration site lies above the filament; (ii) at the same time, we see the appearance of a very small flux rope (SXT, AIA 94 and $131 \AA$ ) and loops \#1 and \#2 associated with the footpoint brightenings north of the filament (AIA $1700 \AA$ A, kernels K1 and K2); and (iii) generation of the $171 \AA$ EUV propagating brightening or disturbances above the filament, which might be associated with the energy release during magnetic reconnection. All these pieces of evidence indicate that the magnetic reconnection took place above the filament.

(3) However, the presence of the filament before the eruption confirms the existence of the flux rope (supporting the filament) before the eruption. Additionally, the reverse J-shaped loop system in the STEREO EUVI $284 \AA$ images also indicates sheared field lines at the eruption site, which were transformed into post-eruptive (cusp-shaped) arcade loops. There are two flux ropes, one supporting the filament that becomes kink unstable, and another one formed above the filament in the sheared arcade loops (observed in the AIA hot channels). Magnetic reconnection above the filament may heat the flux rope to more than about $6 \mathrm{MK}$ and causes it to be visible in the SXT images and AIA hot channels (131 and $94 \AA$ ). The flux rope expanded slowly and developed into a large-scale structure possibly by magnetic reconnection with the surrounding magnetic fields within 15-20 min. Cheng et al. (2014) also reported a similar flux rope structure with twisted or helical threads in hot AIA channels for a different eruptive event. Li \& Zhang (2013) studied the four homologous flux rope structures that appeared during the magnetic reconnection in AIA hot channels. The fourth flux rope (in their observation) was associated with a CME and had a filament underneath that showed counterclockwise rotation and failed to erupt. In our case, we considered the small filament associated with the kink instability (including magnetic reconnection at its footpoint) to cause the onset of the eruption (i.e., by magnetic reconnection above it) and the appearance of a large flux rope above it.

(4) The speed of the flux rope varies between $30-500 \mathrm{~km} \mathrm{~s}^{-1}$. The flux rope does not show significant acceleration (i.e., has a slow rise phase) during the first energy release at about 


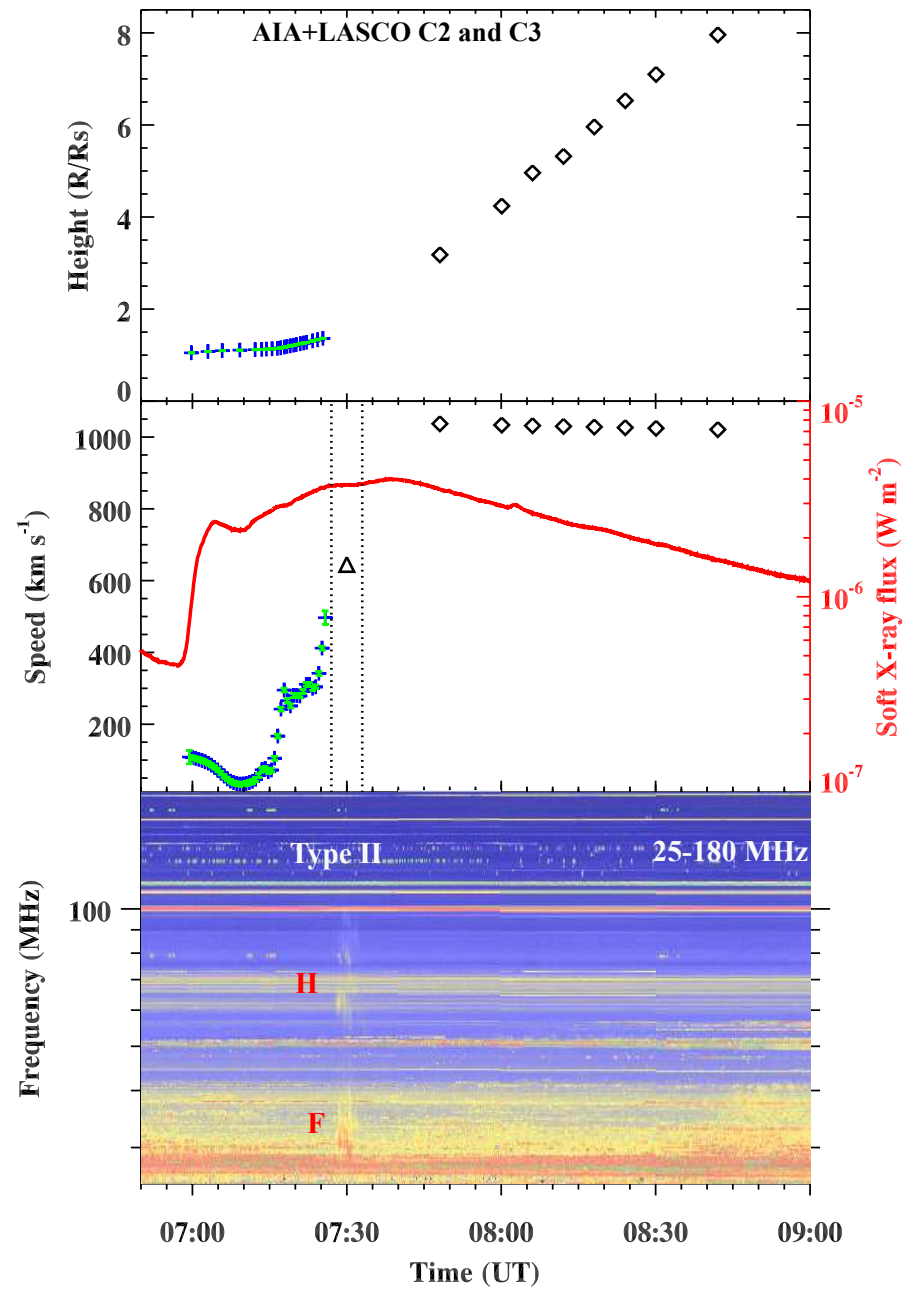

Fig. 12. Top: height-time profile of the flux rope and CME frontal loop (diamond symbol) from AIA $94 \AA$ and LASCO C2/C3. Middle: speed of the flux rope and the CME frontal loop, plotted with GOES soft $\mathrm{X}$-ray profile (red curve). The triangle symbol (between two vertical dotted lines) shows the speed of the type II radio burst excitor. Bottom: dynamic radio spectrum $(25-180 \mathrm{MHz})$ from Learmonth radio observatory. $\mathrm{F}$ and $\mathrm{H}$ represent the fundamental and second harmonic emissions of the type II radio burst.

07:00 UT, but an impulsive acceleration of $\sim 2 \mathrm{~km} \mathrm{~s}^{-2}$ later at 07:14 UT associated with the hard X-ray flux rise and an EUV pulse in $171 \AA$. Moreover, the soft X-ray flux also rises after $\sim 7: 12$ UT. This evidence indicates that the ongoing reconnection in the solar corona results in the acceleration of a large flux rope and the associated particle acceleration from the current sheet underneath the flux rope. In addition, the decrease of the overlying field strength with height also determines the acceleration of the flux rope in the corona by torus instability (Török \& Kliem 2005; Kliem \& Török 2006; Aulanier et al. 2010; Olmedo \& Zhang 2010). However, in our case, magnetic reconnection below the flux rope and the onset of the torus instability at about $1 \times 10^{5} \mathrm{~km}$ height together may cause the impulsive acceleration of the flux rope after 07:12 UT.

Some of the observational features of this event match the prominence eruption studied by Kumar et al. (2012). They observed a breaking of the northern leg of the prominence followed by an unwinding motion (anti-clockwise) caused by the reconnection below the prominence followed by the formation of a helix. However, they observed neither the hard $\mathrm{X}$-ray source $(12-25 \mathrm{keV})$ nor the hot flux rope appearance above the prominence. Probably because of a lack of reconnection above the prominence, which could not heat the flux rope enough for it to be appear in the hot channel. In addition, the prominence successfully erupted and became the core of the CME. Note that here we observed a breaking of the southern leg and unwinding motion (anti-clockwise) in a similar way, but in a smaller filament with magnetic reconnection above it, which caused the appearance of the flux rope and the failed eruption of the filament.

We did not observe the hard X-ray source between the crossing legs of the filament, which may be evidence of a particle acceleration site at the current sheet formed between the crossing legs by the kink instability (Török \& Kliem 2005; Alexander et al. 2006; Cho et al. 2009; Kliem et al. 2010; Joshi et al. 2013). As suggested in the two flux rope model of Gilbert et al. (2001), the magnetic reconnection above the filament (within the flux rope) may cause the failed eruption of the filament, which in turn results in the formation of an inverse-polarity flux rope (new one) above it (middle panel of their Fig. 3). However, the kink instability is not discussed in their model. Very likely, the reconnection above the filament (in our case) suppresses it downward, resulting in the failed eruption (Gilbert et al. 2001, 2007). In addition, our case study demonstrates multiple reconnection above the filament (hard X-ray flux and EUV pulses) that may also cause the sheared magnetic flux to form a large twisted flux rope.

In conclusion, we reported a unique event of a flux rope eruption above a kinked small filament. The magnetic reconnection above the kinked filament is a rare phenomenon, and this type of event has not been observed before. This study highlights the role of multiple reconnection and kink instability in triggering the eruption. Future studies of the similar events using high-resolution multiwavelength data sets will shed more light on the detailed processes involved in these eruptive phenomena related to flux rope formation and eruption.

Acknowledgements. We thank the referee for valuable comments and suggestions to improve the manuscript. SDO is a mission for NASA's Living With a Star (LWS) program. RHESSI is a NASA Small Explorer. Hinode is a Japanese mission developed and launched by ISAS/JAXA, with NAOJ as domestic partner and NASA and STFC (UK) as international partners. It is operated by these agencies in co-operation with ESA and the NSC (Norway). SWAP is a project of the Centre Spatial de Liege and the Royal Observatory of Belgium funded by the Belgian Federal Science Policy Office (BELSPO). SOHO is a project of international cooperation between ESA and NASA. This work was supported by the "Development of Korea Space Weather Center" of KASI and the KASI basic research funds.

\section{References}

Alexander, D., Liu, R., \& Gilbert, H. R. 2006, ApJ, 653, 719

Amari, T., Luciani, J. F., Aly, J. J., Mikic, Z., \& Linker, J. 2003a, ApJ, 585, 1073 Amari, T., Luciani, J. F., Aly, J. J., Mikic, Z., \& Linker, J. 2003b, ApJ, 595, 1231 Amari, T., Aly, J.-J., Mikic, Z., \& Linker, J. 2010, ApJ, 717, L26

Antiochos, S. K., DeVore, C. R., \& Klimchuk, J. A. 1999, ApJ, 510, 485

Aschwanden, M. J., Metcalf, T. R., Krucker, S., et al. 2004, Sol. Phys., 219, 149

Aschwanden, M. J., Nitta, N. V., Wuelser, J.-P., \& Lemen, J. R. 2008, ApJ, 680, 1477

Aulanier, G. 2014, in IAU Symp., 300, 184

Aulanier, G., Török, T., Démoulin, P., \& DeLuca, E. E. 2010, ApJ, 708, 314

Ba̧k-Stȩślicka, U., Gibson, S. E., Fan, Y., et al. 2013, ApJ, 770, L28

Berghmans, D., Hochedez, J. F., Defise, J. M., et al. 2006, Adv. Space Res., 38, 1807 
P. Kumar and K.-S. Cho: Multiwavelength observation of a large-scale flux rope eruption above a kinked small filament

Bothmer, V., \& Schwenn, R. 1998, Annales Geophysicae, 16, 1

Brueckner, G. E., Howard, R. A., Koomen, M. J., et al. 1995, Sol. Phys., 162, 357

Burlaga, L. F., Klein, L., Sheeley, Jr., N. R., et al. 1982, Geophys. Res. Lett., 9, 1317

Canfield, R. C., Hudson, H. S., \& McKenzie, D. E. 1999, Geophys. Res. Lett., 26,627

Chen, P. F. 2011, Liv. Rev. Solar Phys., 8, 1

Chen, P. F., \& Shibata, K. 2000, ApJ, 545, 524

Cheng, X., Zhang, J., Liu, Y., \& Ding, M. D. 2011, ApJ, 732, L25

Cheng, X., Zhang, J., Saar, S. H., \& Ding, M. D. 2012, ApJ, 761, 62

Cheng, X., Ding, M. D., Guo, Y., et al. 2014, ApJ, 780, 28

Cho, K.-S., Lee, J., Bong, S.-C., et al. 2009, ApJ, 703, 1

Démoulin, P., \& Aulanier, G. 2010, ApJ, 718, 1388

Demoulin, P., Henoux, J. C., Priest, E. R., \& Mandrini, C. H. 1996, A\&A, 308, 643

Demoulin, P., Bagala, L. G., Mandrini, C. H., Henoux, J. C., \& Rovira, M. G. 1997, A\&A, 325, 305

Dere, K. P., Brueckner, G. E., Howard, R. A., Michels, D. J., \& Delaboudiniere, J. P. 1999, ApJ, 516, 465

Fan, Y. 2001, ApJ, 554, L111

Fan, Y., \& Gibson, S. E. 2003, ApJ, 589, L105

Fan, Y., \& Gibson, S. E. 2004, ApJ, 609, 1123

Gary, G. A., \& Moore, R. L. 2004, ApJ, 611, 545

Gibson, S. E., Fan, Y., Török, T., \& Kliem, B. 2006, Space Sci. Rev., 124, 131

Gilbert, H. R., Holzer, T. E., \& Burkepile, J. T. 2001, ApJ, 549, 1221

Gilbert, H. R., Alexander, D., \& Liu, R. 2007, Sol. Phys., 245, 287

Golub, L., Deluca, E., Austin, G., et al. 2007, Sol. Phys., 243, 63

Gosling, J. T., Birn, J., \& Hesse, M. 1995, Geophys. Res. Lett., 22, 869

Green, L. M., \& Kliem, B. 2009, ApJ, 700, L83

Green, L. M., Kliem, B., \& Wallace, A. J. 2011, A\&A, 526, A2

Halain, J.-P., Berghmans, D., Defise, J.-M., et al. 2010, in SPIE Conf. Ser., 7732

Hannah, I. G., \& Kontar, E. P. 2013, A\&A, 553, A10

Howard, R. A., Moses, J. D., Vourlidas, A., et al. 2008, Space Sci. Rev., 136, 67

Illing, R. M. E., \& Hundhausen, A. J. 1985, J. Geophys. Res., 90, 275

Illing, R. M. E., \& Hundhausen, A. J. 1986, J. Geophys. Res., 91, 10951

Innes D., Guo, L., Bhattacharjee, A., \& Huang, Y.-M. 2014, ApJ, 796, 27

Ji, H., Wang, H., Schmahl, E. J., Moon, Y.-J., \& Jiang, Y. 2003, ApJ, 595, L135

Joshi, B., Kushwaha, U., Cho, K.-S., \& Veronig, A. M. 2013, ApJ, 771, 1

Karpen, J. T., Antiochos, S. K., \& DeVore, C. R. 2012, ApJ, 760, 81

Kliem, B., \& Török, T. 2006, Phys. Rev. Lett., 96, 255002

Kliem, B., Titov, V. S., \& Török, T. 2004, A\&A, 413, L23

Kliem, B., Linton, M. G., Török, T., \& Karlický, M. 2010, Sol. Phys., 266, 91

Kliem, B., Török, T., \& Thompson, W. T. 2012, Sol. Phys., 281, 137

Kosugi, T., Matsuzaki, K., Sakao, T., et al. 2007, Sol. Phys., 243, 3

Kumar, P., \& Cho, K.-S. 2013, A\&A, 557, A115

Kumar, P., \& Innes, D. E. 2013, Sol. Phys., 288, 255

Kumar, P., Manoharan, P. K., \& Uddin, W. 2010, ApJ, 710, 1195

Kumar, P., Manoharan, P. K., \& Uddin, W. 2011a, Sol. Phys., 271, 149

Kumar, P., Srivastava, A. K., Filippov, B., Erdélyi, R., \& Uddin, W. 2011b, Sol. Phys., 272, 301

Kumar, P., Cho, K.-S., Bong, S.-C., Park, S.-H., \& Kim, Y. H. 2012, ApJ, 746, 67

Kumar, P., Park, S.-H., Cho, K.-S., \& Bong, S.-C. 2013, Sol. Phys., 282, 503

Lemen, J. R., Title, A. M., Akin, D. J., et al. 2012, Sol. Phys., 275, 17
Li, T., \& Zhang, J. 2013, ApJ, 778, L29

Li, X., Morgan, H., Leonard, D., \& Jeska, L. 2012, ApJ, 752, L22

Lin, R. P., Dennis, B. R., Hurford, G. J., et al. 2002, Sol. Phys., 210, 3

Liu, W., Title, A. M., Zhao, J., et al. 2011, ApJ, 736, L13

Longcope, D. W., \& Beveridge, C. 2007, ApJ, 669, 621

Lynch, B. J., Antiochos, S. K., MacNeice, P. J., Zurbuchen, T. H., \& Fisk, L. A. 2004, ApJ, 617, 589

Manoharan, P. K. 2010, Sol. Phys., 265, 137

Manoharan, P. K., van Driel-Gesztelyi, L., Pick, M., \& Demoulin, P. 1996, ApJ, 468, L73

Marubashi, K., Cho, K.-S., Kim, Y.-H., Park, Y.-D., \& Park, S.-H. 2012, J. Geophys. Res. (Space Physics), 117, 1101

Metcalf, T. R., Hudson, H. S., Kosugi, T., Puetter, R. C., \& Pina, R. K. 1996, ApJ, 466, 585

Moore, R. L., Sterling, A. C., Hudson, H. S., \& Lemen, J. R. 2001, ApJ, 552, 833

Okamoto, T. J., Tsuneta, S., Lites, B. W., et al. 2008, ApJ, 673, L215

Olmedo, O., \& Zhang, J. 2010, ApJ, 718, 433

Patsourakos, S., Vourlidas, A., \& Stenborg, G. 2013, ApJ, 764, 125

Pesnell, W. D., Thompson, B. J., \& Chamberlin, P. C. 2012, Sol. Phys., 275, 3

Pevtsov, A. A. 2002, Sol. Phys., 207, 111

Pevtsov, A. A., Canfield, R. C., \& Metcalf, T. R. 1995, ApJ, 440, L109

Priest, E. R., Hood, A. W., \& Anzer, U. 1989, ApJ, 344, 1010

Qiu, J., Hu, Q., Howard, T. A., \& Yurchyshyn, V. B. 2007, ApJ, 659, 758

Rust, D. M., \& Kumar, A. 1994, Sol. Phys., 155, 69

Rust, D. M., \& Kumar, A. 1996, ApJ, 464, L199

Schuck, P. W. 2006, ApJ, 646, 1358

Su, Y., Surges, V., van Ballegooijen, A., DeLuca, E., \& Golub, L. 2011, ApJ, 734,53

Suematsu, Y., Tsuneta, S., Ichimoto, K., et al. 2008, Sol. Phys., 249, 197

Török, T., \& Kliem, B. 2003, A\&A, 406, 1043

Török, T., \& Kliem, B. 2005, ApJ, 630, L97

Török, T., Kliem, B., \& Titov, V. S. 2004, A\&A, 413, L27

Török, T., Chandra, R., Pariat, E., et al. 2011, ApJ, 728, 65

Török, T., Temmer, M., Valori, G., et al. 2013, Sol. Phys., 286, 453

Tripathi, D., Kliem, B., Mason, H. E., Young, P. R., \& Green, L. M. 2009, ApJ, 698, L27

Tsuneta, S., Ichimoto, K., Katsukawa, Y., et al. 2008, Sol. Phys., 249, 167

van Ballegooijen, A. A., \& Martens, P. C. H. 1989, ApJ, 343, 971

van Ballegooijen, A. A., Priest, E. R., \& Mackay, D. H. 2000, ApJ, 539, 983

Vourlidas, A. 2014, Plasma Phys. Controll. Fus., 56, 064001

Vourlidas, A., Lynch, B. J., Howard, R. A., \& Li, Y. 2013, Sol. Phys., 284, 179

Vršnak, B., Maričić, D., Stanger, A. L., \& Veronig, A. 2004, Sol. Phys., 225, 355

Vršnak, B., Maričić, D., Stanger, A. L., et al. 2007, Sol. Phys., 241, 85

Webb, D. F., \& Howard, T. A. 2012, Liv. Rev. Sol. Phys., 9, 3

Wuelser, J.-P., Lemen, J. R., Tarbell, T. D., et al. 2004, in Telescopes and Instrumentation for Solar Astrophysics, eds. S. Fineschi, \& M. A. Gummin, SPIE Conf. Ser., 5171, 111

Yan, X. L., Qu, Z. Q., Kong, D. F., \& Xu, C. L. 2012, ApJ, 754, 16

Yashiro, S., Gopalswamy, N., Michalek, G., et al. 2004, J. Geophys. Res. (Space Physics), 109, 7105

Yurchyshyn, V. 2008, ApJ, 675, L49

Zhang, J., \& Dere, K. P. 2006, ApJ, 649, 1100

Zhang, J., Dere, K. P., Howard, R. A., \& Vourlidas, A. 2004, ApJ, 604, 420

Zhang, J., Cheng, X., \& Ding, M.-D. 2012, Nat. Commun., 3 